\title{
The Most Oxygen-Poor Planetary Nebula
}

\author{
Michael G. Richer, Gaghik Tovmassian \\ Observatorio Astronómico Nacional, Instituto de Astronomía, UNAM, \\ P.O. Box 439027, San Diego, CA 92143-9027, USA \\ Grażyna Stasińska \\ Observatoire de Meudon, 5 Place Jules Janssen, Meudon Cedex, \\ F-92195, France \\ Carlos Gutierrez \\ Instituto de Astrofísica de Canarias, Vía Láctea, s/n, La Laguna, \\ Tenerife, Canary Islands E-38200, Spain \\ Christian Veillet \\ Canada-France-Hawaii Telescope Corp., P.O. Box 1597, Kamuela, HI \\ 96743, USA
}

\begin{abstract}
PNG135.9+55.9 is a halo planetary nebula recently discovered among the objects from the Second Byurakan Survey. Based upon our first observations, a photoionization model analysis shows that the oxygen abundance in the nebula must be extremely low, probably $10^{-2}$ $10^{-3}$ of the solar value. This finding has strong implications on our understanding of the formation of planetary nebulae, of mixing processes in the stellar progenitors and of the evolution of the Galactic halo.
\end{abstract}

\section{Discussion}

Recently, PN G 135.9+55.9 has been recognized as a halo planetary nebula (PN; renamed from SBS $1150+599 \mathrm{~A}$ ) that is extremely oxygen-poor (Tovmassian et al 2001), with an $\mathrm{O} / \mathrm{H}$ abundance ratio between $10^{-2}$ and $10^{-3}$ of the solar value. It is the most oxygen-poor PN known so far, and its oxygen abundance is similar to the lowest measured in stars to date (e.g., Boesgaard et al 1999).

The original optical spectra (4000-7000 $\AA$ ) show only the Balmer lines of hydrogen, He II $\lambda \lambda 4686,5411$, and very weak [O III] $\lambda 5007$ ( 4\% of $\mathrm{H} \beta$; Tovmassian et al 2001). The emission lines are blue shifted, having a heliocentric radial velocity of about $-200 \mathrm{~km} / \mathrm{sec}$, easily compatible with a location in the galactic halo. The very blue continuum is dominated by a stellar component.

Our subsequent spectroscopic observations confirmed the previous findings, particularly the absence of $\mathrm{He}$ I and other low ionization state lines. In addition, they provide a measure of the [Ne III] $\lambda 3869$ intensity and useful upper limits to the $[\mathrm{Ne} \mathrm{V}] \lambda 3425$ and [O II] $\lambda 3727$ intensities. We also obtained narrow-band 


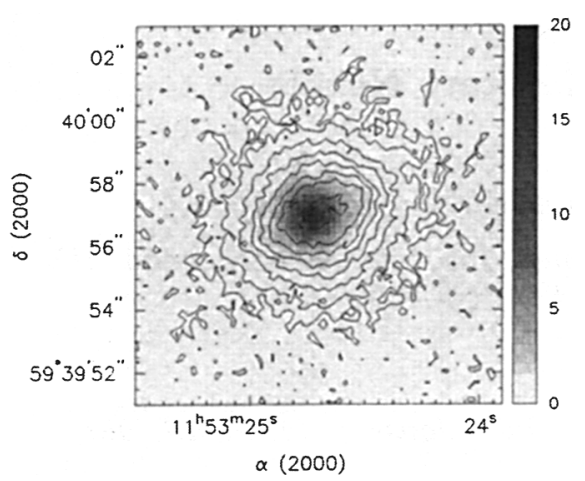

Figure 1. This figure shows our continuum image of PN G $135.9+55.9$ with the $\mathrm{H} \alpha$ contours overlaid.

images in $\mathrm{H} \alpha$ and the nearby continuum, which confirmed the extended nature of the nebular emission and the presence of a very hot exciting star (Fig. 1).

We find $\mathrm{Ne} / \mathrm{O}$ around 0.7 , i.e., about 3 times larger than in $\mathrm{PNe}$ of the Galactic disk and similar to the value found for the peculiar halo PN BB1 (Howard et al. 1997). This implies that either the $\mathrm{Ne} / \mathrm{O}$ ratio in $\mathrm{PN} \mathrm{G}$ $135.9+55.9$ was modified by its progenitor star, or the interstellar $\mathrm{Ne} / \mathrm{O}$ prior to the formation of the progenitor of PN G 135.9+55.9 had a non-standard value, perhaps implying an inhomogeneous early Galactic halo.

PN G 135.9+55.9 is an interesting probe of the pregalactic helium abundance, since it is strongly density bounded and helium is seen only as $\mathrm{He}^{++}$. We find $\mathrm{He} / \mathrm{H}$ in the range found for extragalactic metal-poor $\mathrm{H}$ II regions by Olive et al (1997) and Izotov et al (1999) (in preparation). One complication is that some of the He might be "missing" in PN G 135.9+55.9, having been converted into $\mathrm{C}$ by the triple- $\alpha$ reaction.

Upcoming ultraviolet observations of PN G 135.9+55.9 with FUSE will measure the ultraviolet $\mathrm{N}$ III and $\mathrm{C}$ III lines, complementing the abundances derived from ground-based observations to include $\mathrm{H}, \mathrm{He}, \mathrm{C}, \mathrm{N}, \mathrm{O}$, and $\mathrm{Ne}$, and better constrain the ionization structure of the nebula. Once the $\mathrm{C}$ abundance is known, it will be possible to evaluate how much pregalactic helium it might lock up.

\section{References}

Boesgaard, A.M., King, J.R., Deliyannis, C.P., \& Vogt, S.S, 1999, AJ, 117, 492

Howard, J.W., Henry, R. B. C., \& McCartney, S., 1997, MNRAS, 284, 465

Izotov, Y.I. et al 1999, ApJ 527, 757

Olive, K.A., Steigman, G., \& Skillman, E.D., 1997, ApJ 483, 788

Tovmassian, G.H. et al 2001, A\&A, 370, 456 